\section{OC28 HOW DOES PRIMARY CHILD HEALTH CARE FARE IN EUROPE? - A JOURNEY INTO THE UNKNOWN}

${ }^{1}$ Mitch Blair*, 'Denise Alexander, ${ }^{1}$ Michael Rigby. 'Imperial College, London, UK; ${ }^{2}$ Trinity College, Dublin, Israel

10.1136/archdischild-2019-epa.27

Introduction/Background Children are a fifth of Europe's population; they are citizens, future workers, future parents and carers, and the future elderly population. However they are dependent on society meeting their specific needs for effective primary care, which provides some $90 \%$ of all health contact, yet there is little research into how well current services do this.

Methods The MOCHA project was funded by the EU as a Horizon 2020 project to appraise models of child primary health care in all 30 EU/EEA countries. The 20 multidisciplinary scientific partners used local agents, networks, and literature to assess structural, cultural, sociological and political dimensions. An External Advisory Board assisted the project; partners from Australia, Switzerland and the USA gave global context. The appraisal was based on a holistic multi stakeholder framework correlating structure, process and outcome measures. 81 children from 5 countries were interviewed. A number of tracer conditions spanning primary care prevention (immunisation and screening), chronic (asthma) and complex care (long term ventilation, ADHD and intractable epilepsy) were selected to help illustrate the systems in place.

Results Direct attribution of positive outcome measures of morbidity and mortality to primary care separate from wider health system factors (finance, workforce capacity/competence) proved impossible due to lack of comparable data. A number of optimum design principles emerged.

MOCHA reached six Conclusions:

1. Primary care for children in each country comprises many components; their cohesion as a system is determined more by their accessibility, capacity, and relationship than by their style (such as general or paediatrician primary care practitioner).

2. Effectiveness is primarily determined by access, workforce, service coordination and continuity, inter-sectoral governance, sociocultural linkage, and financing.

3. Optimal primary care for children is child-centric, equitable, proactive, integrated with specialist, social care and education services, and based on (and yielding) robust evidence.

4. Interdependence of health, economy and society is more influential than system construct, but there is inadequate public health, primary care and inter-sectoral collaboration on child health and development concerns.

5. Children are unacceptably invisible in health data and policy in Europe, including rights definition, data sets, research activity, e-health, and policy innovation.

6. Focussed cross-Directorate and inter-agency activity within Europe would strengthen evidence and policy to facilitate stronger national systems.
RESPIRATORY SYNCYTIAL VIRUS HOSPITAL ADMISSION AND OUTCOME IN IRELAND AMONG INFANTS BORN LESS THAN 32 WEEKS GESTATION IN THE ERA OF CHANGING PALIVIZUMAB PRESCRIPTION GUIDELINES

Karen McCarthy*, Eilis Moore, Karina Butler, Ronan Leahy, Patrick Gavin. Rainbow Paediatric Infectious Disease Department, Our Ladies Children's Hospital, Crumlin, Dublin, Ireland

\subsection{6/archdischild-2019-epa.28}

Background Mortality and morbidity among children with Respiratory Syncytial Virus (RSV) bronchiolitis is higher among infants born prematurely and those with chronic lung or congenital heart disease. Palivizumab is a monoclonal antibody that provides passive immunity to RSV and can modify the disease course in high-risk infants. In 2006, the American Academy of Paediatrics published a clinical practice guideline recommending that infants born less than 32-weeks gestation receive Palivizumab if aged less than twelve months at the start of the RSV season. An updated guideline in 2014 however, advised that only infants less than 29-weeks should receive Palivizumab in the absence of other indications. The aim of this study was to determine the impact of changing guidelines on RSV-related hospital admissions, management and outcome among infants born $<32$ weeks in Ireland.

Methods Data was collected prospectively for all patients admitted with RSV bronchiolitis from 2004-2017 at a tertiary paediatric hospital. The two groups of interest were infants born <32 weeks gestation admitted from 2004-2014 and from 2014-2018. Data included demographic variables, background, management and outcome. Categorical variables were analysed using Chi-squared test. Continuous variables were analysed using Mann-Whitney test.

Results There were 2851 admissions with RSV-bronchiolitis during the study period. There were 39 infants $<32$ weeks admitted from 2004-2014 (2.4\% total) and 28 from 20142018 (2.3\%). Compliance with Palivizumab prescription guidelines was $53.8 \%$ pre-2014 and $78.5 \%$ post-2014 among hospitatilised infants. The proportion of paediatric intensive care unit (PICU) admissions in infants born $<32$ weeks gestation reduced by $20.7 \%$ and there was a $36 \%$ reduction in the requirement for mechanical ventilation in the post-2014 cohort (p 0.002). Among infants born 29-32 there were fewer PICU admissions, less ventilator support required $(\mathrm{p}=0.007)$ and more humidified high flow nasal cannula oxygen therapy (HHFNC) used ( $p$ 0.005). The use of nebuised saline and salbutamol significantly reduced ( $\mathrm{p} 0.01)$. The median length of hospital admission was reduced by 6 days $(0.015)$.

Conclusion The 2014 updated AAP-guidelines on Palivizumab use has been associated with reduced prescription in the 2932 week gestation group. This change has not resulted in increased frequency of hospital admission or PICU admission among infants $<32$ weeks gestation. In fact, PICU admissions reduced and the need for mechanical ventilation was significantly less. Furthermore, we have seen an improved evidencebased medicine approach to the management of these infants and a $50 \%$ reduction in median length of hospital stay. 